\title{
Characterization on Responsiveness of Excitatory Synaptic Transmissions to a1-Adrenoceptor Blockers in Substantia Gelatinosa Neurons Isolated From Lumbo-Sacral Level in Rat Spinal Cords
}

\author{
Daisuke Uta ${ }^{1,2, *}$, Tsuyoshi Hattori ${ }^{3, *}$, Megumu Yoshimura ${ }^{2,4,5}$ \\ ${ }^{1}$ Department of Applied Pharmacology, Graduate School of Medicine and Pharmaceutical Sciences, University of Toyama, Toyama, Japan \\ ${ }^{2}$ Department of Integrative Physiology, Graduate School of Medical Sciences, Kyushu University, Fukuoka, Japan \\ ${ }^{3}$ Department of Medical Affairs, Asahi Kasei Pharma Co., Tokyo, Japan \\ ${ }^{4}$ Graduate School of Health Sciences, Kumamoto Health Science University, Kumamoto, Japan \\ ${ }^{5}$ Nogata Nakamura Hospital, Fukuoka, Japan
}

Purpose: The aim of this study was to characterize the responsiveness of miniature excitatory postsynaptic currents (mEPSCs) to a1-adrenoceptor blockers in substantia gelatinosa (SG) neurons from the spinal cord to develop an explanation for the efficacy of al-adrenoceptor blockers in micturition dysfunction.

Methods: Male adult Sprague-Dawley rats were used. Blind whole-cell patch-clamp recordings were performed using SG neurons in spinal cord slices. Naftopidil $(100 \mu \mathrm{M})$, tamsulosin $(100 \mu \mathrm{M})$, or silodosin $(30 \mu \mathrm{M})$, al-adrenoceptor blockers, was perfused. The frequency of mEPSCs was recorded in an SG neuron to which the 3 blockers were applied sequentially with wash-out periods. Individual frequencies in a pair before naftopidil and tamsulosin perfusion were plotted as baseline, and the correlation between them was confirmed by Spearman correlation coefficient; linear regression was then performed. The same procedure was performed before naftopidil and silodosin perfusion. Frequencies of pairs after naftopidil and tamsulosin perfusion and after naftopidil and silodosin perfusion were similarly analyzed. The ratios of the frequencies after treatment to before were then calculated.

Results: After the treatments, Spearman $\rho$ and the slope were decreased to 0.682 from 0.899 at baseline and 0.469 from 1.004 at baseline, respectively, in the tamsulosin group relative to the naftopidil group. In the silodosin group, Spearman $\rho$ and the slope were also decreased to 0.659 from 0.889 at baseline and 0.305 from 0.989 at baseline, respectively, relative to the naftopidil group. Naftopidil significantly increased the ratio of the frequency of mEPSCs compared to tamsulosin and silodosin $(\mathrm{P}=0.015$ and $\mathrm{P}=0.004$, respectively).

Conclusions: There was a difference in responsiveness in the frequency of mEPSCs to $\alpha 1$-adrenoceptor blockers, with the response to naftopidil being the greatest among the al-adrenoceptor blockers. These data are helpful to understand the action mechanisms of a1-adrenoceptor blockers for male lower urinary tract symptoms in clinical usage.

Keywords: Adrenergic alpha1 antagonists; Excitatory postsynaptic currents; Naftopidil; Substantia gelatinosa

Corresponding author: Daisuke Uta (iD https://orcid.org/0000-0001-5644-1348 Department of Applied Pharmacology, Graduate School of Medicine and Pharmaceutical Sciences, University of Toyama, Toyama 930-0194, Japan E-mail: daicarp@pha.u-toyama.ac.jp / Tel: +81-76-434-7513 / Fax: +81-76-434-5045

Submitted: February 22, 2019 / Accepted after revision: March 10, 2019

*Daisuke Uta and Tsuyoshi Hattori contributed equally to this study as co-first authors. 
- Grant/Fund Support: This work was supported by grants from the programs for Grants-in-Aid for Scientific Research (DU and MY) of the Ministry of Education, Science, Sports and Culture of Japan (grant numbers JP15K08667, JP25860431, JP23790652, JP25460733, JP21600005), and was partially conducted by the MEXT-Supported Program for the Strategic Research Foundation at Private Universities, "Creation of 3D nano-micro structures and its application to biomimetics and medicine”, and 2015-2019 (DU, grant number S1511031). This study was also supported in part by the Asahi Kasei Pharma Corporation.

- Research Ethics: All experiments were performed in accordance with the "Guiding Principles for Care and Use of Animals in the Field of Physiological Sciences" of the Physiological Society of Japan and were approved by the local Animal Experiment Committee of the Kumamoto Health Science University and Kyushu University.

- Conflict of Interest: This study was also supported in part by the Asahi Kasei Pharma Corporation. Except for that, no potential conflict of interest relevant to this article was reported.

\section{- HIGHLIGHTS}

- To analyze mEPSC responsiveness to a 1-adrenoceptor blockers, patch-clamp technique was performed.

- The differences in mEPSC responsiveness between naftopidil and other blockers were large.

- The ratio of the frequency was larger in the naftopidil group than in the tamsulosin and silodosin groups.

\section{INTRODUCTION}

Lower urinary tract symptoms (LUTS) consist of voiding, storage, and postmicturition symptoms [1]. Male LUTS is related to a variety of causes, e.g., benign prostatic obstruction/benign prostatic hyperplasia, bladder dysfunction including overactive bladder, and nocturnal polyuria [2]. The prevalence of LUTS increases with aging, and a considerable proportion of men are affected by LUTS [3]. To manage male LUTS pharmacologically, several kinds of drugs have been used, such as $\alpha 1$-adrenoceptor blockers, 5 '-reductase inhibitors, phosphodiesterase 5 inhibitors, and plant extracts. Among them, al-adrenoceptor blockers are the most established drugs, and they have been taken generally and widely. The a1-adrenoceptor blockers reduce enhanced tonus or contractility in the urethra and prostate by relaxation of smooth muscle, thus increasing the lowered urine flow rates [4], which is a representative voiding symptom.

In addition, $\alpha 1$-adrenoceptors have also been investigated to improve storage symptoms [5], and a mechanism of action has been studied in animal studies. In conscious cystometry using rats, tamsulosin, naftopidil, and silodosin prolonged the micturition interval [6]. Tamsulosin, BMY7378, and silodosin were also studied in conscious rats, in which each blocker prolonged intercontraction intervals with cerebroventricular injection, but only tamsulosin and silodosin lengthened the intervals when added intrathecally [7]. When isovolumetric cystometry was conducted with an anesthetic, naftopidil increased intercontraction intervals significantly, but tamsulosin weakly or did not increased [8]. In an ex vivo study using detrusor strips, naftopidil suppressed contractility in the control and bladder outlet obstruction rats, but not tamsulosin, silodosin, and prazosin
[9]. The differences were thought to be based on selectivity for receptor subtypes of a1-adrenoceptors and others, but the rationale remains to be clarified and is unconvincing.

By using voltage-clamp recordings, it is possible to identify primary afferent information in substantia gelatinosa (SG, lamina II of Rexed) [10] neurons mediated by different primary afferent fibers $[11,12]$. The efficacy of synaptic transmission is determined by presynaptic transmitter release probability and postsynaptic responsiveness. Analyses of frequency and amplitude distributions of miniature excitatory postsynaptic currents (mEPSCs) permit us to determine the loci of experimental manipulation (i.e., presynaptic and/or postsynaptic) [13]. Recently, it was reported that naftopidil suppressed the amplitude of evoked EPSCs (eEPSCs), which are activated by dorsal root stimuli from afferent fibers, and naftopidil appeared to suppress the micturition reflex [14]. However, the effects of a1-adrenoceptor blockers on an excitatory synaptic current at synaptic terminal sites in the spinal cord remained to be determined, although an inhibitory synaptic current has already been investigated [15].

Recently, naftopidil prolonged intercontraction intervals with intrathecal injection in rats [16] and facilitated the frequency of miniature inhibitory post synaptic currents in SG neurons from lumbo-sacral levels of the spinal cord in rats using a patch clamp technique $[14,15]$. The former effects of naftopidil were antagonized by intrathecal bicuculine, a GABA (type-A $\gamma$-aminobutyric acid) receptor antagonist, and/or strychnine, a glycine receptor antagonist, and the latter effects were also attenuated by bicuculine and strychnine. Moreover, naftopidil decreased the amplitude of eEPSCs in SG neurons by inputs from primary afferent neurons from the spinal cord in rats, but prazosin did not $[14,15]$. As EPSCs show glutamatergic 
transmission in afferent fibers, decreasing EPSCs are related to inhibition of micturition [17]. This study focused on EPSCs at a synaptic terminal site, and mEPSCs were measured and analyzed in SG neurons. In addition, the effects of the a1-adrenoceptor blockers naftopidil, tamsulosin, and silodosin on mEPSCs in SG neurons from the lumbo-sacral spinal cord in rats were investigated using patch-clamp recording to develop an explanation for the diversity of efficacy in micturition dysfunction of a1-adrenoceptor blockers.

\section{MATERIALS AND METHODS}

\section{Spinal Cord Slice Preparation}

The methods for obtaining slices of the adult rat spinal cord and for blind patch-clamp recordings from SG neurons have been described in detail elsewhere $[14,15,18,19]$. Briefly, adult male Sprague-Dawley rats (6-8 weeks old) were deeply anaesthetized with urethane $(1.2 \mathrm{~g} / \mathrm{kg}$, intraperitoneally), and a lumbosacral laminectomy was then performed. The lumbosacral segments of the spinal cord (L2-S3) with ventral and dorsal roots were removed and placed in ice-cold Krebs solution equilibrated with $95 \% \mathrm{O}_{2}-5 \% \mathrm{CO}_{2}$. The Krebs solution contained (in $\mathrm{mM}$ ): $\mathrm{NaCl} 117, \mathrm{KCl} 3.6, \mathrm{CaCl}_{2} 2.5, \mathrm{MgCl}_{2}$ 1.2, $\mathrm{NaH}_{2} \mathrm{PO}_{4}$ 1.2, NaH$\mathrm{CO}_{3} 25$, and glucose $11(\mathrm{pH}, 7.4)$. Immediately after removal of the spinal cord, the rats were killed by exsanguination under urethane anesthesia. The pia-arachnoid membrane was removed after cutting all the ventral and dorsal roots. The spinal cord was mounted on a vibratome, and a $500-\mu \mathrm{m}$-thick transverse slice with the attached dorsal root was cut. The slice was placed on a nylon mesh in the recording chamber in a volume of 0.5-mL Krebs solution, and it was completely submerged and perfused with Krebs solution saturated with 95\% $\mathrm{O}_{2}$ and 5\% $\mathrm{CO}_{2}$ at $37^{\circ} \mathrm{C} \pm 1^{\circ} \mathrm{C}$ and a flow rate of $10-15 \mathrm{~mL} / \mathrm{min}$.

\section{Whole-Cell Patch Clamp Recordings From SG Neurons}

The SG was easily discernible with transmitted illumination as a relatively translucent band across the dorsal horn in the transverse slice preparations (Fig. 1A). Blind whole-cell voltage clamp recordings were made from SG neurons, as previously described $[14,15,19,20]$. The patch pipettes were filled with a solution containing potassium gluconate solution (in $\mathrm{mM}$ ): $\mathrm{K}$ gluconate 135, $\mathrm{KCl}$ 5, $\mathrm{CaCl}_{2}$ 0.5, $\mathrm{MgCl}_{2}$ 2, EGTA 5, HEPES 5, and ATP-Mg 5 ( $\mathrm{pH}, 7.2)$. The tip resistance of the patch pipettes was 6-12 $\mathrm{M} \Omega$. Series resistance was assessed according to the response to a $5-\mathrm{mV}$ hyperpolarizing step. This value was monitored during the recording session, and data were rejected if values changed by $>15 \%$. Signals were acquired with a patch clamp amplifier (Axopatch 700A, Molecular Devices, Union City, CA, USA). The data were digitized with an analog to digital/digital to analog converter (Digidata 1321A, Molecular Devices), stored on a personal computer using a data acquisition program (Clampex version 9.0, Molecular Devices), and analyzed using a software package (Clampfit version 9.0, Molecular Devices). Cell recordings were made in voltage-clamp mode at holding potentials of $-70 \mathrm{mV}$ to record EPSCs $[14,15,19]$.

\section{Drug Application}

1-[4-(2-methoxyphenyl) piperaznyl]-3-(1-naphthyloxy) propan-2-ol (naftopidil) (PubChem CID: 4418) (Asahi Kasei Pharma Co., Tokyo, Japan) was dissolved in 1\% dimethyl sulf-

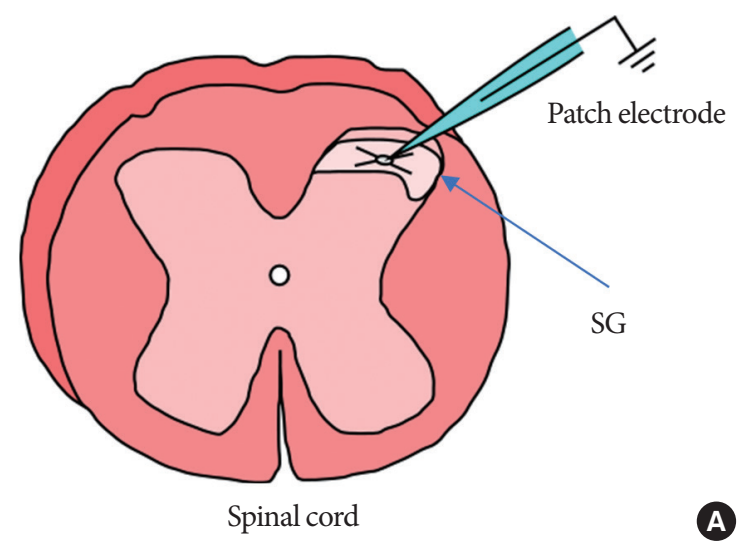

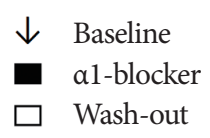

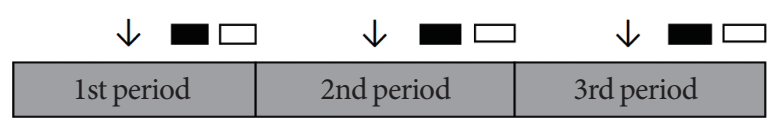

mEPSC recording

Fig. 1. The experimental method and design are illustrated. (A) Blind whole-cell patch-clamp recording is set in a substantia gelatinosa (SG) neuron of an adult rat spinal dorsal horn. (B) In a single neuron, three al-adrenoceptor blockers are evaluated sequentially with wash-out periods. 
oxide (PubChem CID: 679) (Wako, Osaka, Japan) in Krebs solution. Tamsulosin and silodosin were dissolved in Krebs solution. The concentration of naftopidil and tamsulosin used were $100 \mu \mathrm{M}$, and silodosin used was $30 \mu \mathrm{M}$. Since naftopidil $(100 \mu \mathrm{M})$ was effective in modulating synaptic transmission in superficial dorsal horn neurons of rodent spinal cord slices [15], naftopidil was used at $100 \mu \mathrm{M}$ in the present study. Although, we used same concentration of tamsulosin $(100 \mu \mathrm{M})$, we could not prepare the same concentration of silodosin. However, the affinity of the receptor of silodosin is 100 times higher than naftopidil [21], so we considered $30 \mu \mathrm{M}$ silodosin is sufficient concentration. All drugs were applied by perfusion sequentially in a single cell with wash-out periods via a 3-way stopcock without changes in the perfusion rate or temperature. The application schedule is summarized in Fig. 1B.

\section{Statistical Analysis}

Frequency is expressed as median (25\%-75\% tile), because the distribution pattern of the individual data did not show normality. Individual frequencies for the pair of before naftopidil and before tamsulosin treatment were plotted as a baseline, and the correlation between them was confirmed by Spearman correlation coefficient; linear regression by the least squares meth- od was then performed to assess the coefficient of determination and slope. A similar analysis was performed for the pair of before naftopidil and before silodosin treatment. In addition, frequencies for the pairs of after naftopidil and tamsulosin treatment and after naftopidil and silodosin treatment were analyzed as described above. The ratios of frequencies after to before treatment were compared among the a1-adrenoceptor blockers by the Steel-Dwass test using JMP ver. 14 (SAS Institute Inc., Cary, NC, USA). When the changes of the ratios were $+20 \%$ or more and $-20 \%$ or less, they were defined as positive and negative responses, respectively [14,19]. Finally, the proportions of positive and negative changes were calculated for each a1-adrenoceptor blocker.

\section{RESULTS}

\section{Characteristics of the Baseline Frequencies of mEPSCs Before Treatment With Each a1-Adrenoceptor Blocker}

In the analyses of the baseline frequencies of mEPSCs, the range of the medians was 10.5 to $10.8 \mathrm{~Hz}$ for the three a1adrenoceptor blockers (Table 1). Between the naftopidil and tamsulosin groups and between the naftopidil and silodosin groups, Spearman $\rho$ values for the baseline frequency of mEP-

Table 1. Characteristics of the baseline frequencies of miniature excitatory postsynaptic currents before the treatment of each a1adrenoceptor blocker

\begin{tabular}{lccr}
\hline al-Adrenoceptor blocker & Naftopidil $(\mathrm{Hz})$ & Tamsulosin $(\mathrm{Hz})$ & Silodosin $(\mathrm{Hz})$ \\
\hline Median & 10.5 & 10.6 & 10.8 \\
$25 \%$ and $75 \%$ tiles & $(4.3-12.4)$ & $(5.0-13.0)$ & $(5.6-13.6)$ \\
Range & $(1.0-31.1)$ & $(1.1-31.6)$ & $(1.8-31.4)$ \\
\hline
\end{tabular}

The baseline frequencies were recorded before perfusion of each al-adrenoceptor blocker and after the wash-out period $(\mathrm{N}=36)$.
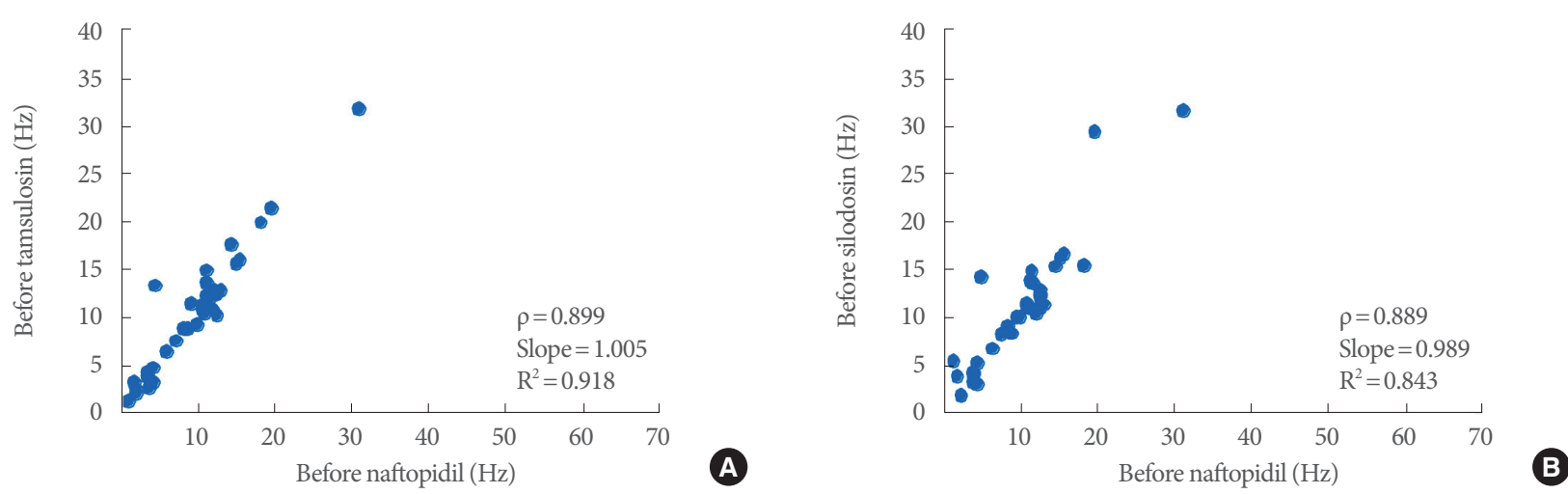

Fig. 2. Correlations of the frequencies of miniature excitatory postsynaptic current at baseline. (A) Before naftopidil treatment vs. before tamsulosin treatment. (B) Before naftopidil treatment vs. before silodosin treatment. $\mathrm{N}=36$. 
SCs were 0.899 and 0.889 , respectively. The baseline frequencies of mEPSCs in the tamsulosin group or the silodosin group to the naftopidil group were plotted in a linear fashion, with slopes of 1.004 and 0.989 , respectively (Fig. 2A, B). The values of $\mathrm{R}^{2}$ in the regressions were 0.918 for tamsulosin versus naftopidil and 0.843 for silodosin versus naftopidil.

\section{Relationships of $\alpha 1$-Adrenoceptor Blockers to the Frequency of mEPSCs}

The effects of a1-adrenoceptor blockers on the frequencies of mEPSCs are shown as typical traces (Fig. 3A-C). After treatment in the tamsulosin group relative to the naftopidil group, the values of Spearman $\rho$ and the slope were decreased to 0.682 from 0.899 at baseline and to 0.469 from 1.005 at baseline, re- spectively (Fig. 3D). After treatment in the silodosin group, the values of Spearman $\rho$ and the slope were also decreased to 0.659 from 0.889 at baseline and to 0.295 from 0.989 at baseline compared to the naftopidil group (Fig. 3E). Naftopidil significantly increased the ratio of the frequencies of mEPSCs compared to tamsulosin and silodosin ( $\mathrm{P}=0.015$ and $\mathrm{P}=0.004$, respectively) (Fig. 4).

A total of $33 \%, 17 \%$, and $17 \%$ of SG neurons tested responded to naftopidil, tamsulosin, and silodosin, respectively. The number (proportion) of SG neurons with positive responsiveness to naftopidil, tamsulosin, and silodosin were 9 neurons (25\%), 4 neurons (11\%), and 4 neurons (11\%) in the total of 36 neurons, respectively (Table 2, Fig. 5). The number with negative responsiveness by naftopidil, tamsulosin, and silodosin
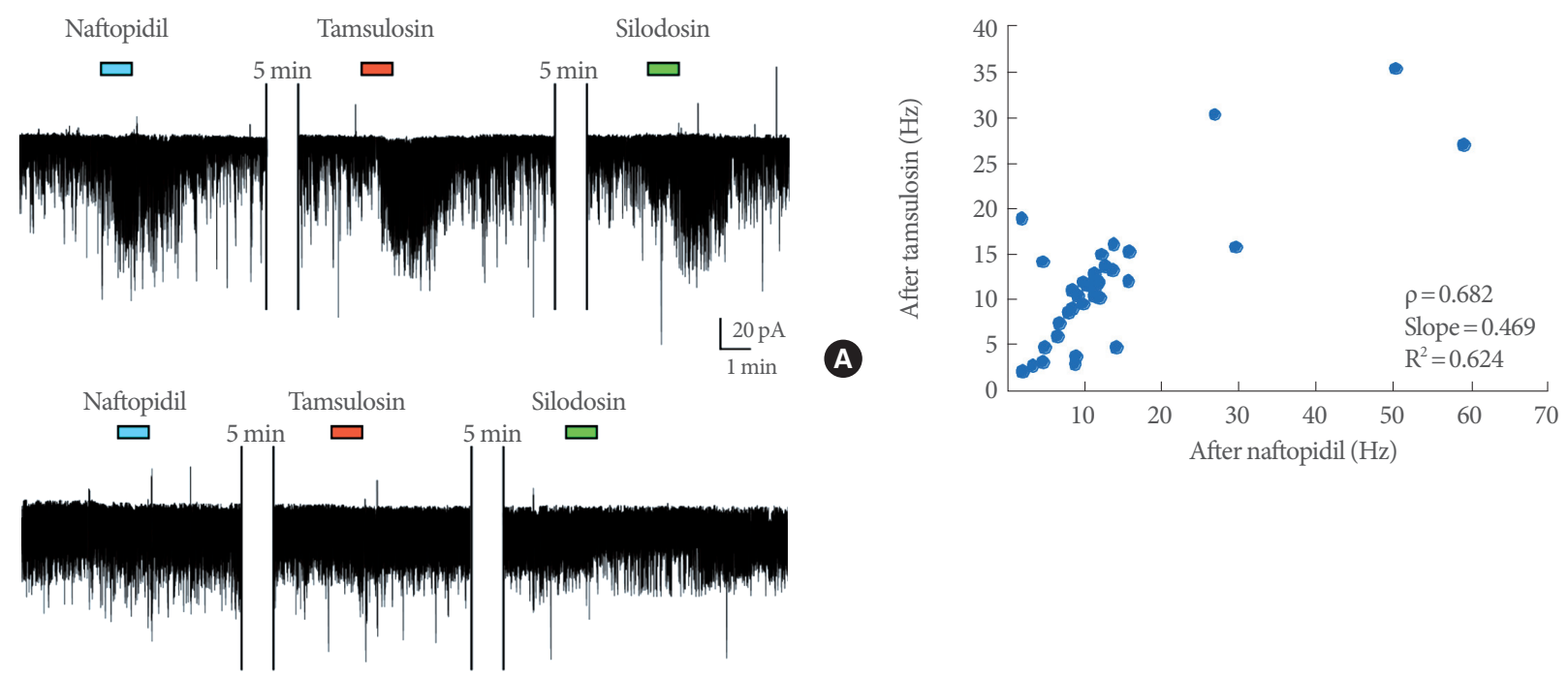

(D)
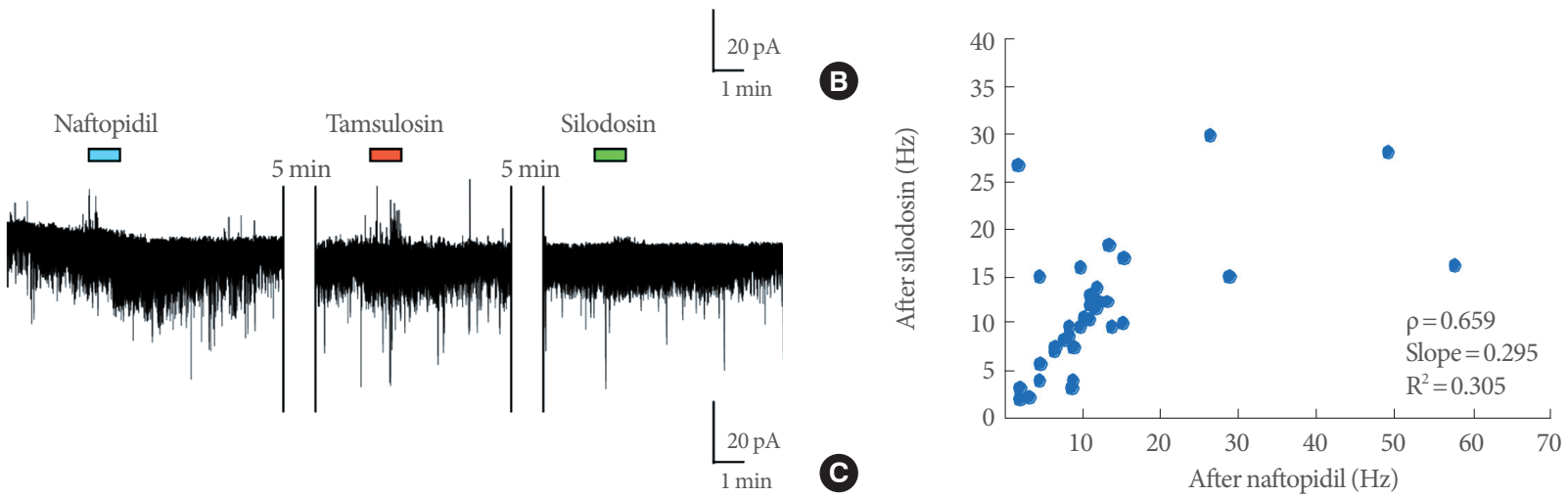

Fig. 3. The effects of $\alpha 1$-adrenoceptor blockers on miniature excitatory postsynaptic current (mEPSCs). (A-C) Typical charts presenting the responses to three $\alpha 1$-adrenoceptor blockers, response to no $\alpha 1$-adrenoceptor blockers, and response to only naftopidil, respectively. Color bars show durations of drugs. (D, E) Correlation of the frequencies of mEPSCs after naftopidil and tamsulosin treatment, and after naftopidil and silodosin treatment, respectively. $\mathrm{N}=36$. 
Table 2. A number of substantia gelatinosa neurons responded during the treatment of each $\alpha 1$-adrenoceptor blocker

\begin{tabular}{lccc}
\hline al-Adrenoceptor blocker & Naftopidil & Tamsulosin & Silodosin \\
\hline Positive response & $9(25)$ & $4(11)$ & $4(11)$ \\
Negative response & $3(8)$ & $2(6)$ & $2(6)$ \\
No response & $24(67)$ & $30(83)$ & $30(83)$ \\
Subtotal & $36(100)$ & $36(100)$ & $36(100)$ \\
\hline
\end{tabular}

Value are presented as number (\%) of substantia gelatinosa neurons.

After the baseline recording, each al-adrenoceptor blocker was applied. There were wash-out periods between terms perfusing al-adrenoceptor blockers.

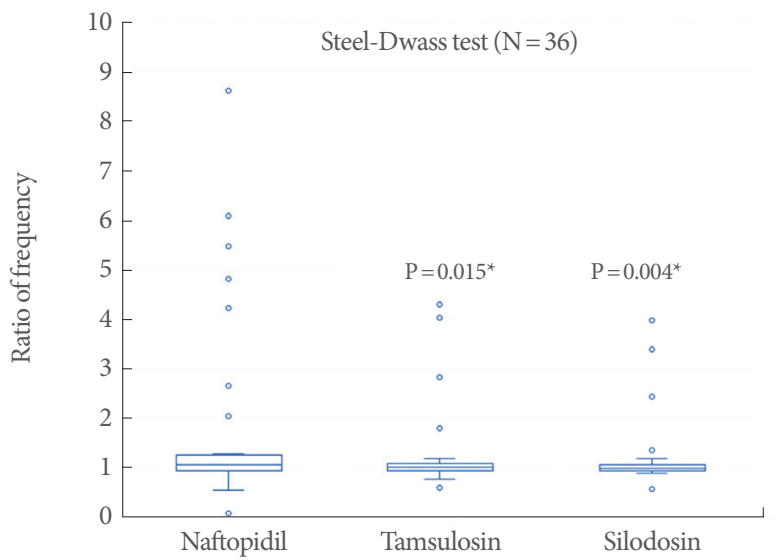

Fig. 4. Ratios of the frequencies after to before treatment. ${ }^{\star} \mathrm{Com}-$ pared with naftopidil.

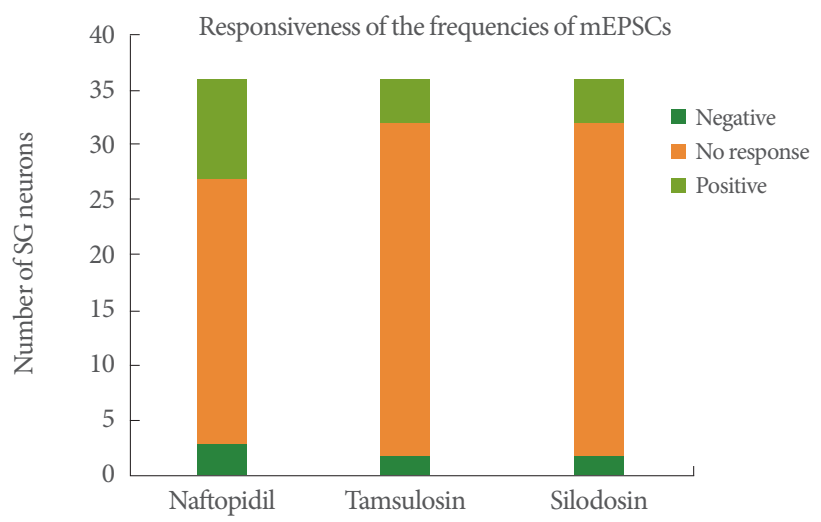

Fig. 5. The ratios of posttreatment with each al-adrenoceptor blocker to baseline for the frequencies of miniature excitatory postsynaptic current (mEPSCs). SG, substantia gelatinosa.

were 3 neurons (8\%), 2 neurons (6\%), and 2 neurons $(6 \%)$ in the total of 36 neurons, respectively. The characteristics of SG neurons with respect to responsiveness to those al-adrenoceptor blockers are summarized in Fig. 6. Three neurons responded to all a1-adrenoceptor blockers. No neurons responded to tamsu-

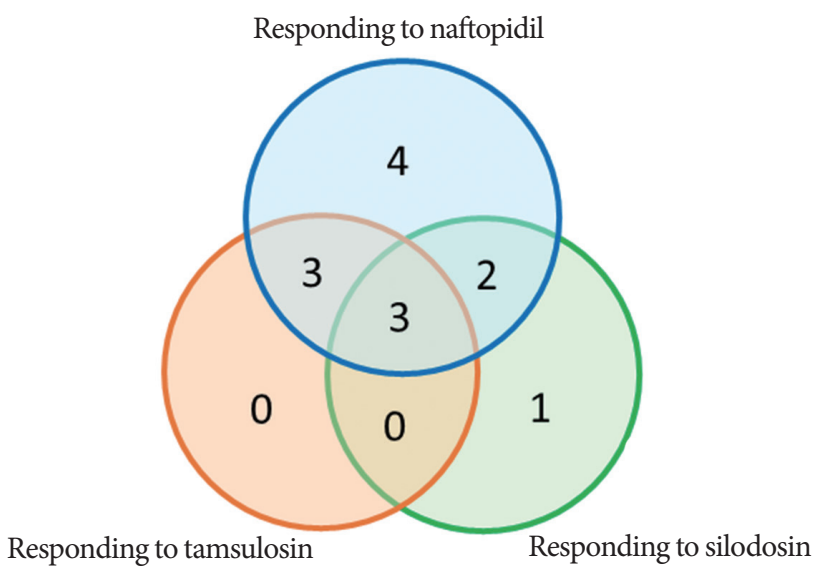

No response to any drugs: 23 neurons

Fig. 6. Summary of the responsiveness of the frequencies of miniature excitatory postsynaptic current to al-adrenoceptor blockers.

losin alone or tamsulosin and silodosin. Only one neuron was observed to respond to silodosin alone.

\section{DISCUSSION}

In a single SG neuron, three al-adrenoceptor blockers were sequentially evaluated with mEPSC recording by patch-clump technique, and the frequencies of mEPSCs were analyzed. Various types of responsiveness to each al-adrenoceptor blocker were observed. In particular, the differences in responsiveness between naftopidil and other al-blockers were remarkable, as shown by the proportion of response. The ratio of the frequency after treatment to that at baseline was significantly larger in the naftopidil group than in the tamsulosin and silodosin groups.

\section{Baseline Characteristics of the Frequency of mEPSCs}

In the relationship between the pair of before naftopidil and 
tamsulosin treatments, the correlation coefficient was strong. Since the values of $\mathrm{R}^{2}$ and slope of the regression line were 0.918 and 1.005, respectively, the baseline value in the tamsulosin group was fairly estimated by that in the naftopidil group, and the baseline values of both groups were virtually identical to each other. The relationship between another pair of before naftopidil and silodosin treatments was similarly considered. These results suggest that the baseline frequencies of MEPSCs were comparable among the groups, and the repeated recordings were reproducible. Therefore, the changes in frequency could be used to directly compare the a1-adrenoceptor blockers.

\section{Responsiveness of the Frequency of mEPSCs to a1-Adrenoceptor Blockers}

If the correlation of frequencies of mEPSCs between the naftopidil and tamsulosin groups was comparable after treatment with them, the value of the slope in the regression line for the frequencies ought to be around 1.000. In contrast, the slope of the regression line after treatment was 0.469 , far smaller than that at baseline. Furthermore, the correlation coefficient of the frequencies between the naftopidil and tamsulosin groups was weaker than that at baseline. These results strongly indicate that the responsiveness of the SG neuron to naftopidil differs from that to tamsulosin. This was similar for the pair of silodosin and naftopidil. These findings are contrasted with the reproducibility of the experiment of eEPSCs with repeated treatment of naftopidil [14]. The ratio of the frequency after treatment of naftopidil to that at baseline was significantly higher than for both tamsulosin and silodosin. The proportion of responses in the naftopidil group showed a different trend from the other groups (Fig. 5). These results suggest that naftopidil acts uniquely among these a1-adrenoceptor blockers in the spinal cord. This is supported by the previous study that demonstrated that naftopidil decreased the amplitude of eEPSCs, but prazosin did not [14].

\section{Extrapolation of Changes of the mEPSCs to the Micturition Reflex}

The SG (lamina II) of the spinal dorsal horn contains a high density of excitatory and inhibitory interneurons that are thought to be critically involved in the modulation of nociception [22]. Facilitation of the frequency of mEPSCs is understood as an increase in glutamate release from presynaptic terminals and upregulation of receptors for glutamate at synaptic terminal sites [22], resulting in activation of inhibitory or excitatory interneurons. Since the present study was not performed chronically in normal animals, upregulation of the receptor should be excluded. In the previous study, glutamatergic excitatory transmission was found in the descending limb of the spinobulbospinal micturition reflex pathway [23], and glutamatergic mechanisms play an essential role for micturition in the rat spinal cord [24]. In the pelvic-urethra reflex, N-methyl-d-aspartate-dependent potentiation is inhibited via spinal $\gamma$-aminobutyric acidergic (GABAergic) inhibition [25]. Intrathecal injection of a1adrenoceptor blockers clearly inhibits the micturition reflex in animal models $[7,8]$. The effect of intrathecal naftopidil on the inhibition of the micturition reflex is antagonized by bicuculine and/or strychnine; although each intrathecal bicuculine or strychnine injection partially suppresses the abolished isovolumetric bladder contraction caused by naftopidil, the combination injection of bicuculine and strychnine completely inhibits the effect of naftopidil [16]. Therefore, these studies may support the idea that the effects of a1-adrenoceptor blockers, particularly naftopidil, on increases of the frequency of mEPSCs indicate facilitation of inhibitory interneurons, such as GABAergic and glycinergic neurons.

Differences among a1-adrenoceptor blockers with respect to the degree of efficacy in male LUTS have been controversial. Gotoh et al. reported that naftopidil is as effective as tamsulosin on the symptom basis using the International Prostate Symptom Score [26,27]. Nishino et al. [28] reported that maximum desired volume and first desired volume were higher with naftopidil than with tamsulosin in a pressure flow study. In 2 of 7 patients, involuntary contractions were disappeared by naftopidil, but not tamsulosin. Silodosin showed greater improvement in overactive bladder symptoms along with the urinary flow rate in patients with benign prostatic enlargement complicated by overactive bladder than naftopidil [29]. Silodosin reduced urologic symptom scores, similar to other a1-adrenoceptor blockers [30]. Although these discrepancies obviously depend on the study design, the differences should be noted on the basis of the responsiveness of SG neurons to a1-adrenoceptor blockers (Fig. 6).

\section{Multimodal Responsiveness of the Frequencies to a1-Adrenoceptor Blockers}

In the present study, not every SG neuron responded to $\alpha 1$ adrenoceptor blockers based on the frequency of mEPSCs. The low rates are not unusual, since a total of 20\%-30\% SG neurons responded in a previous study, investigating cinnamaldehyde and/or capsaicin [19]. As shown in Fig. 5, there seem to be 3 
types of SG neurons in responsiveness of the mEPSC frequency to a1-adrenoceptor blockers, being positive, deficient responsiveness, and negative. The population showing positive response may be considered to activate inhibitory interneurons such as GABAergic and glycinergic neurons, while the negative population possibly suppresses inhibitory inputs to inhibitory neurons. It is not surprising that part of the SG neurons responded to a1-adrenoceptor blockers. The reason why is that nociceptive stimulant and sensory irritation, including pain and the urge to void, are modulated by inter neurons in multiple and complex way [31]. The low responsiveness of SG neurons to a1-adrenoceptor blockers may mean that they play one role in the complex system.

In conclusion, there were differences in responsiveness of the frequencies of mEPSCs to al-adrenoceptor blockers. Naftopidil showed the greatest effect among the a1-adrenoceptor blockers evaluated. These results indicate that all $\alpha 1$-adrenoceptor blockers do not have same effects. The difference possibly means that usefulness of a1-adrenoceptor blockers is different from each other patients. In the management of male LUTS, the present results are helpful to understand the presence of patients, who have different reactivity to al-adrenoceptor blockers, and the action mechanisms of a1-adrenoceptor blockers for LUTS in clinical usage. Also, the current study may present a rationale to make decision like a change of prescribe when the drug shows minor potency for patients suffer from male LUTS. In addition, the frequency of mEPSCs may be a unique target for LUTS, particularly a micturition reflex.

This study has some limitations. Although afferent nerves from urothelium largely project to lamina $\mathrm{X}$ in the dorsal horn, SG neurons in lamina II were used. As described previously, part of the superficial neurons receives nociceptive and non-nociceptive inputs from the lower urinary tract, showing upregulation of cFos expression [32]. Although using lamina $\mathrm{X}$ is ideal, it has a low density of neurons, and the low density is inconvenient for blindly attaching patch-clamp electrodes. Therefore, lamina II was examined. In the future, the afferent input needs to be examined directly from the lower urinary tract in vivo.

\section{AUTHOR CONTRIBUTION STATEMENT}

- Full access to all the data in the study and takes responsibility for the integrity of the data and the accuracy of the data analysis: $D U$

-Study concept and design: $D U, M Y$
- Acquisition of data: $D U$

- Analysis and interpretation of data: $D U, T H$

- Drafting of the manuscript: $D U, T H$

- Critical revision of the manuscript for important intellectual content: $M Y$

- Statistical analysis: $D U, T H$

- Obtained funding: $M Y$

- Administrative, technical, or material support: TH

-Study supervision: $M Y$

\section{REFERENCES}

1. Abrams P, Cardozo L, Fall M, Griffiths D, Rosier P, Ulmsten U, et al. The standardisation of terminology of lower urinary tract function: report from the Standardisation Sub-committee of the International Continence Society. Neurourol Urodyn 2002;21:167-78.

2. Management of non-neurogenic male LUTS [Internet]. Arnhem (The Netherlands): EAU Guideline Office [cited 2018 Dec 8]. EAU Guidelines. Edn. presented at the EAU Annual Congress Copenhagen 2018. ISBN 978-94-92671-01-1. Available from: http://uroweb.org/ guideline/treatment-of-non-neurogenic-male-luts/?type=archive.

3. Rohrmann S, Katzke V, Kaaks R. Prevalence and progression of lower urinary tract symptoms in an aging population. Urology 2016; 95:158-63.

4. Takei R, Ikegaki I, Shibata K, Tsujimoto G, Asano T. Naftopidil, a novel alpha1-adrenoceptor antagonist, displays selective inhibition of canine prostatic pressure and high affinity binding to cloned human alpha1-adrenoceptors. Jpn J Pharmacol 1999;79:447-54.

5. Kwon SY, Lee KS, Yoo TK, Chung JI, Lee JY, Hong JH, et al. Comparison of the effect of naftopidil $75 \mathrm{mg}$ and tamsulosin $0.2 \mathrm{mg}$ on the bladder storage symptom with benign prostatic hyperplasia: prospective, multi-institutional study. Urology 2018;111:145-50.

6. Chen Z, Ishizuka O, Imamura T, Aizawa N, Igawa Y, Nishizawa O, et al. Role of alpha1-adrenergic receptors in detrusor overactivity induced by cold stress in conscious rats. Neurourol Urodyn 2009; 28:251-6.

7. Yoshizumi M, Matsumoto-Miyai K, Yonezawa A, Kawatani M. Role of supraspinal and spinal alphal-adrenergic receptor subtypes in micturition reflex in conscious rats. Am J Physiol Renal Physiol 2010;299:F785-91.

8. Sugaya K, Nishijima S, Miyazato M, Ashitomi K, Hatano T, Ogawa Y. et al. Effects of intrathecal injection of tamsulosin and naftopidil, alpha-1A and -1D adrenergic receptor antagonists, on bladder activity in rats. Neurosci Lett 2002 2;328:74-6.

9. Sakai T, Kasahara K, Tomita K, Ikegaki I, Kuriyama H. Naftopidil 
inhibits 5-hydroxytryptamine-induced bladder contraction in rats. Eur J Pharmacol 2013;700:194-200.

10. REXED B. The cytoarchitectonic organization of the spinal cord in the cat. J Comp Neurol 1952;96:414-95.

11. Yang K, Kumamoto E, Furue H, Li YQ, Yoshimura M. Action of capsaicin on dorsal root-evoked synaptic transmission to substantia gelatinosa neurons in adult rat spinal cord slices. Brain Res 1999;830:268-73.

12. Yang K, Feng Y, Li Y. Baclofen inhibition of dorsal root-evoked inhibitory postsynaptic currents in substantia gelatinosa neurons of rat spinal cord slice. Brain Res 2001;900:320-3.

13. Yang K, Li YQ. Origins of spontaneous and noxious stimuli-evoked miniature EPSCs in substantia gelatinosa. Neuroreport 2001;12:3942.

14. Uta D, Hattori T, Yoshimura M. Effects of high concentrations of naftopidil on dorsal root-evoked excitatory synaptic transmissions in substantia gelatinosa neurons in vitro. Int Neurourol J 2018; 22:252-9.

15. Uta D, Xie DJ, Hattori T, Kasahara KI, Yoshimura M. Effects of naftopidil on inhibitory transmission in substantia gelatinosa neurons of the rat spinal dorsal horn in vitro. J Neurol Sci 2017;380:205-11.

16. Sugaya K, Nishijima S, Kadekawa K, Ashitomi K, Ueda T, Yamamoto H. Spinal mechanism of micturition reflex inhibition by naftopidil in rats. Life Sci 2014;116:106-11.

17. Guo YX, Li DP, Chen SR, Pan HL. Distinct intrinsic and synaptic properties of pre-sympathetic and pre-parasympathetic output neurons in Barrington's nucleus. J Neurochem 2013;126:338-48.

18. Yoshimura M, Jessell TM. Primary afferent-evoked synaptic responses and slow potential generation in rat substantia gelatinosa neurons in vitro. J Neurophysiol 1989;62:96-108.

19. Uta D, Furue H, Pickering AE, Rashid MH, Mizuguchi-Takase H, Katafuchi T, et al. TRPA1-expressing primary afferents synapse with a morphologically identified subclass of substantia gelatinosa neurons in the adult rat spinal cord. Eur J Neurosci 2010;31:196073.

20. Yoshimura M, Nishi S. Blind patch-clamp recordings from substantia gelatinosa neurons in adult rat spinal cord slices: pharmacological properties of synaptic currents. Neuroscience 1993;53:51926.

21. Tatemichi S, Tomiyama Y, Maruyama I, Kobayashi S, Kobayashi K, Maezawa A, et al. Uroselectivity in male dogs of silodosin (KMD3213), a novel drug for the obstructive component of benign prostatic hyperplasia. Neurourol Urodyn 2006;25:792-9.
22. Todd AJ. Neuronal circuitry for pain processing in the dorsal horn. Nat Rev Neurosci 2010;11:823-36.

23. Matsumoto G, Hisamitsu T, de Groat WC. Non-NMDA glutamatergic excitatory transmission in the descending limb of the spinobulbospinal micturition reflex pathway of the rat. Brain Res 1995; 693:246-50.

24. Yoshiyama M, de Groat WC. Supraspinal and spinal alpha-amino3-hydroxy-5-methylisoxazole-4-propionic acid and N-methyl-Daspartate glutamatergic control of the micturition reflex in the urethane-anesthetized rat. Neuroscience 2005;132:1017-26.

25. Chen SL, Huang YH, Kao YL, Chen GD, Cheng CL, Peng HY, et al. Acute anal stretch inhibits NMDA-dependent pelvic-urethra reflex potentiation via spinal GABAergic inhibition in anesthetized rats. Am J Physiol Renal Physiol 2008;295:F923-31.

26. Gotoh M, Kamihira O, Kinukawa T, Ono Y, Ohshima S, Origasa H, et al. Comparison of tamsulosin and naftopidil for efficacy and safety in the treatment of benign prostatic hyperplasia: a randomized controlled trial. BJU Int 2005;96:581-6.

27. Griwan MS, Karthikeyan YR, Kumar M, Singh BJ, Singh SK. Comparative evaluation of naftopidil and tamsulosin in the treatment of patients with lower urinary tract symptoms with benign prostatic hyperplasia. Urol Ann 2014;6:181-6.

28. Nishino Y, Masue T, Miwa K, Takahashi Y, Ishihara S, Deguchi T. Comparison of two alpha1-adrenoceptor antagonists, naftopidil and tamsulosin hydrochloride, in the treatment of lower urinary tract symptoms with benign prostatic hyperplasia: a randomized crossover study. BJU Int 2006;97:747-51.

29. Matsukawa Y, Funahashi Y, Takai S, Majima T, Ogawa T, Narita H, et al. Comparison of silodosin and naftopidil for efficacy in the treatment of benign prostatic enlargement complicated by overactive bladder: a randomized, prospective study (SNIPER Study). J Urol 2017;197:452-8.

30. Jung JH, Kim J, MacDonald R, Reddy B, Kim MH, Dahm P. Silodosin for the treatment of lower urinary tract symptoms in men with benign prostatic hyperplasia. Cochrane Database Syst Rev 2017;11:CD012615.

31. Todd AJ. Identifying functional populations among the interneurons in laminae I-III of the spinal dorsal horn. Mol Pain 2017;13: 1744806917693003.

32. Birder LA, de Groat WC. Increased c-fos expression in spinal neurons after irritation of the lower urinary tract in the rat. J Neurosci 1992;12:4878-89. 